\title{
Diabetes Might Augment the Severity of COVID-19: A Current Prospects
}

\author{
Jayeeta Sur ${ }^{1}$, Juhi Sharma ${ }^{2}$ and Divakar Sharma ${ }^{3 *}$ \\ ${ }^{1}$ Independent Researcher, Kolkata, India, ${ }^{2}$ Department of Botany and Microbiology, St. Aloysius College, Jabalpur, India, \\ ${ }^{3}$ Central Research Facility (CRF), Mass Spectrometry Laboratory, Kusuma School of Biological Sciences, Indian Institute of \\ Technology Delhi, New Delhi, India
}

Keywords: diabetes, COVID-19, SARS-CoV-2, hyperglyceamia, ACE2

\section{INTRODUCTION}

COVID-19 is the recently emerged outbreak across the globe which is caused by SARS-CoV-2 (enveloped, positive single-stranded RNA virus). To date, more than 49.7 million confirmed cases and more than 1.2 million deaths have been reported worldwide (10 November 2020), making COVID-19 a major human health concern (1). Predominantly, SARS-CoV-2 infects lower airways and creates respiratory as well as systemic illness which further progresses to a severe form of pneumonia-like conditions in $10-15 \%$ of patients (2). SARS-CoV-2 can infect people of any age, but old-age people as well as people with comorbidities like chronic obstructive pulmonary disease (COPD), cerebrovascular disease, coronary heart disease, hypertension, tuberculosis, tobacco smoking, and diabetes are under a higher risk to get SARS-CoV-2 infection and develop COVID-19. Various reports suggested that these comorbidities might potentiate the COVID-19 progression and severities (3-5); therefore, researchers get attention toward this direction and try to manage this situation.

Various studies revealed that among the cases of COVID-19 with comorbidities, $12-22 \%$ cases have diabetes, and the mortality rate in these cases is 6-7\% (3-6). In this opinion, we have to provide a summary of the symptomatic characteristics of COVID-19 with diabetes, and critical assessment of the association between COVID-19 and diabetes. Therefore, this opinion article could provide meaningful insight for future research and contribute to better clinical management of diabetes patients with COVID-19.

\section{COULD DIABETES POTENTIATE COVID-19}

Recently, a report by Yang and colleagues has shown (4) that out of a group of 52 ICU patients with COVID-19, the distinct comorbidities of 32 non-survivors were cerebrovascular diseases $(22 \%, 7$ cases) and diabetes $(22 \%, 7$ cases). Similarly, a study of 1,099 patients with confirmed COVID-19 showed that out of 1,099 cases, 173 were the case of comorbidities of which $16.2 \%$ were diabetic (3). A study also showed that out of 140 patients admitted being COVID-19 positive, $12 \%$ of patients had diabetes (5). These studies have shown that COVID-19 patients with diabetes are treated with angiotensin-converting enzyme (ACE) inhibitors, but their treatments were not assessed. China's largest epidemiological investigation also showed COVID-19 mortality with diabetes up to $7.3 \%(80 / 1,102)$, which is dramatically higher than that of patients without any comorbidity $(0.9 \%, 133 / 15,536)(7)$. Kulscar et al., through an in vivo experiment using type 2 diabetic transgenic mouse models that express the DDP-4 receptor on pulmonary alveolar cells, have studied the effect of diabetes on MERS-coronavirus infection and its severity. They observed a remarkable association of diabetes with greater weight loss and pulmonary inflammation having macrophage infiltration as similar to the clinical disease (8). 
The most striking observation is that the mortality rate in men is higher than in women diabetic patients who were infected by SARS-CoV-2 (4). Men are more affected by COVID-19 as compared to women, which are probably due to three times higher expression of ACE2 in men than women (9). In an experiment, the continuous infusion of angiotensin 1-7 has shown a vasodilator effect only in female rats (10). The main reason behind this observation is that women are less severely affected by COVID-19 than men which might be due to the protective effect of estrogenmediated ACE2 regulation in premenopausal women (11). A study showed the potential influence of menstrual status and sex hormones on females infected with SARS-CoV-2 and suggested menopause, an independent risk factor for female COVID-19 patients. Estradiol (E2) is a protective factor and regulates the cytokine-related immune responses in premenopausal women (12). Therefore, post-menopausal women lose the protective effects of estrogens so they have a potential risk for type2 diabetes; hence, this comorbidity definitely increases the risk for COVID-19. Recommended drugs for diabetes and associated comorbidities like pioglitazone, glucagon-like peptide1 agonists, statins, diuretics, and mineralocorticoid inhibitors increase the expression of ACE2 level (13). It has been shown that angiotensin-converting enzyme inhibitors (ACEIs) and angiotensin-receptor blockers (ARBs) increase the ACE2 expression level in the brain, heart, and kidney (11).

\section{ROLE OF ACE/ACE2 IN DIABETES AND COVID-19}

The angiotensin-converting enzyme (ACE) is secreted in the lungs and kidneys by the endothelium (inner layer) of blood vessels that regulate the volume of the fluids in the body and hence control blood pressure. ACE is the main component of the renin-angiotensin system (RAS) and converts angiotensinI (ANGI) to the active angiotensin-II (ANGII). ANGII further increases blood pressure (BP) and inflammation, which causes tissue injury as well as increases damage to blood vessel linings. Angiotensin-converting enzyme 2 (ACE2) is also an important element of the RAS pathway which counteracts the effects of ANGII. It is reported that ACE2 expressed by epithelial cells of the lung, kidney, intestine, and blood vessels helps pathogenic coronaviruses such as SARS-CoV and SARS-CoV-2 in their target cell binding and endocytosis (14).

ACE2 is considered as the host cell surface receptor that interacts directly with the spike glycoprotein (S protein) of SARSCoV-2 (3). A recent study has been suggested that ACE2 has 1020 times higher binding affinity to the receptor-binding domain (RBD) of SARS-CoV-2 than SARS-CoV (15). Spike proteins of SARS-CoV-2 bind to host ACE2, so theoretically the normal functioning of ACE2 is imbalanced which makes the aggravation of ANGII effect, which may further cause inflammation, death of alveoli cells, and tissue injury especially to the heart and lungs in COVID-19 patients.
After consideration of the facts that the utilization of ACE2 binds the spike of SARS-CoV-2 and after further endocytosis $(16,17)$, it led to the reduction of ACE2 availability which resulted in the overactivation of RAS (18). Therefore, this overactivation of RAS could be responsible for the adverse risk of COVID-19 in patients with diabetes. In this regard, the application of RAS inhibitors in patients with COVID-19 with pre-existing diabetes could be a potential therapeutic option.

It is evident from a study that entry of SARS-CoV-2 to the host cell depends on spike-ACE2 interaction and this can be blocked by transmembrane serine protease-2 (TMPRSS2) inhibitors because TMPRSS2 has been used for spike (S) protein priming by SARS-CoV-2 (19). It also indicates that the antibodies against SARS-CoV could partially protect against SARS-CoV2 infection. These outcomes have significant implications to understanding SARS-CoV-2 transmissibility and pathogenesis and reveal therapeutic intervention targets.

A significant increase of the ACE2 expression in type 1 or type 2 diabetic patients has been reported while they are treated with ACEIs and ARBs (14). This report suggested that patients with diabetes were treated with drugs that increased the ACE2 expression, so such patients could be at a higher risk of SARS-CoV-2 infection and severity of COVID-19. However, other meta-analysis suggested no significant association between the use of ACEIs or ARBs with the risk of SARS-CoV-2 infection and severity of COVID-19 in hypertensive patients and other old-age patients (6) which is just opposite to COVID-19 with diabetes comorbidities (14). Still, more research is needed to support these contradictory facts. Therefore, these COVID-19 patients with comorbidities should be monitored for ACE2-modulating medications, like ACEIs and ARBs (20).

At low intracellular $\mathrm{pH}, \mathrm{SARS}-\mathrm{CoV}-2$ spikes bind to the host ACE2 enzyme and enter into the cell, causing the infection $(21,22)$. Diabetes may have a strong association with low intracellular $\mathrm{pH}$ and could potentiate the SARS-CoV-2 infection. SARS-CoV-2, ACEIs, and ARBs bind to ACE2 which can further increase the level of ANGII. The increased level of ANGII can activate the $\mathrm{Na}^{+} / \mathrm{H}^{+}$exchanger (NHE) which further reduces the intracellular $\mathrm{pH}$. NHE pumps $3 \mathrm{Na}^{+}$ions into the cell and $2 \mathrm{H}^{+}$ions out of the cell (21). Apart from that, it also transports $\mathrm{Ca}^{2+}$ ions inward the cell. Overall transports of ions make the intracellular environment acidic and hypoxic, which leads to the production of reactive oxygen species (ROS) and cell death. NHA2 is the isoform of the $\mathrm{Na}^{+} / \mathrm{H}^{+}$exchanger (NHE) present in the beta cells of the pancreas and involved in the secretion of insulin (23). In diabetic patients, continuous activation of NHA2 through SARS-CoV-2 infection and a higher level of ANGII lead to pancreatic beta-cell injury via the overproduction of ROS. Therefore, sustainable activation of NHA2 in COVID-19 and diabetes coinfection can damage the pancreatic beta cells or tissue of the pancreas permanently and lead to disease severity as well as death. Consequently, the use of ACEIs and ARBs in diabetes patients can lead to increased morbidity and COVID-19 mortality. 


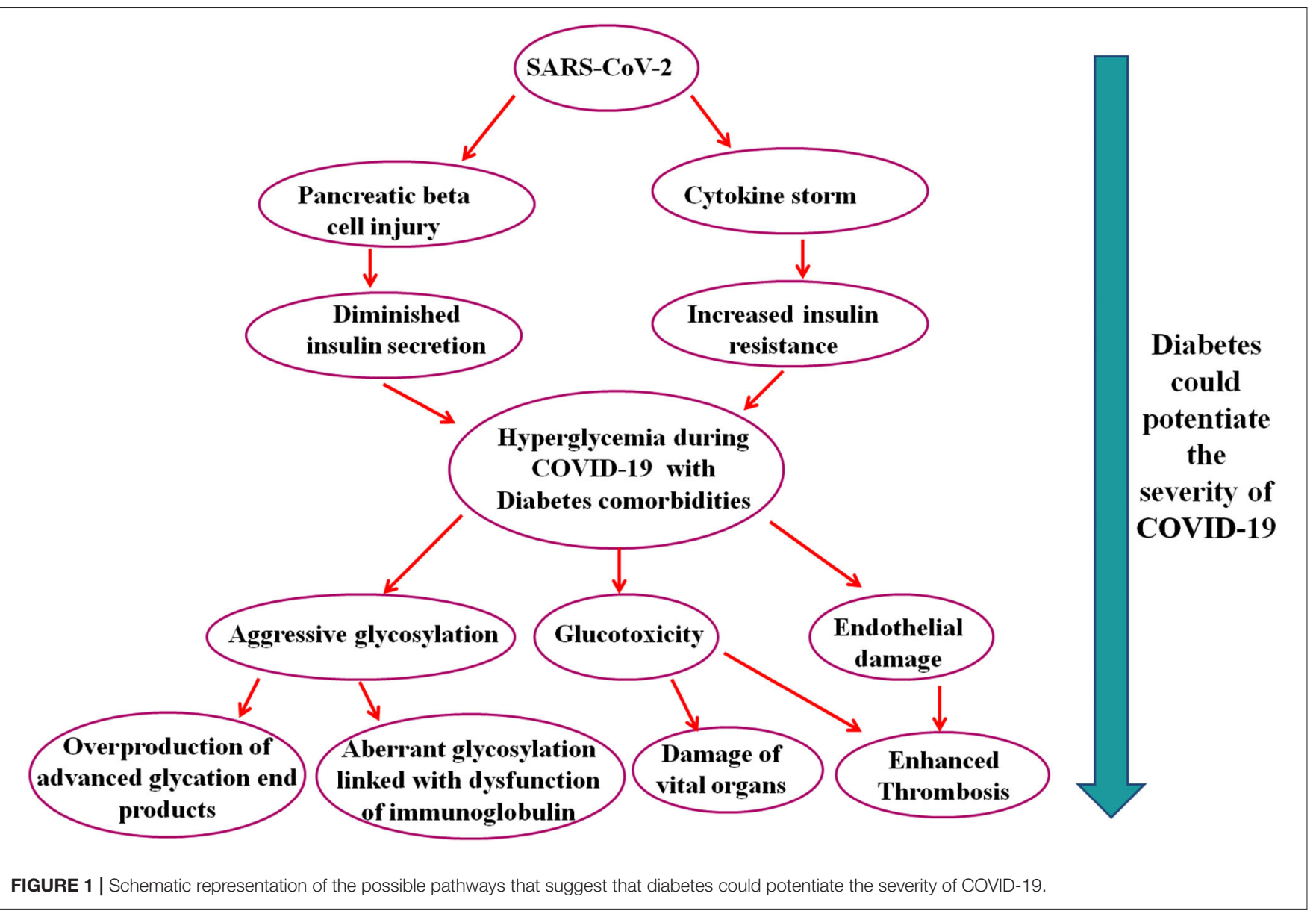

\section{CYTOKINE STORM AND BIOMARKER LEVEL IN DIABETIC PATIENTS DUE TO COVID-19}

Inflammatory cytokine secretion may occur due to immune system imbalance. The cytokine is released from white blood cells and transmits signals from one immune cell to another especially during the entry of unwanted invaders. When cytokines are released in large volume due to hyperactivity of the immune cells, this phenomenon is called a cytokine storm (24). Higher levels of pro-inflammatory cytokines are also observed in COVID-19 patients (25). Data from Chinese studies showed that diabetic patients with COVID-19 displayed higher IL6 as well as C-reactive protein and cause severe pulmonary infection and death. Cytokine storm is the major key factor for this pulmonary infection and death $(3-5,26)$. In diabetes patients, hyperglycemia stimulates the synthesis of cytokines. Some inflammation-related biomarkers like IL-6, serum ferritin, ESR, and CRP are present in higher concentrations in diabetic patients and linked to the disease-related cytokine storm. Among them, IL-6 is a very good predictor for disease prognosis and severity because its expression time is longer than other cytokines such as TNF and IL-1 $(3,5)$. In addition to this, a significant amount in serum ferritin indicates the activation of the monocyte-macrophage system, which is crucial for an inflammatory storm (27). These results indicate that patients with diabetes-COVID-19 coinfection are more susceptible to formation of an inflammatory storm, which eventually leads to rapid deterioration of COVID-19.

A study showed that urea nitrogen, albumin, and N-terminal probrain natriuretic peptide (NT-proBNP) level increased in diabetes with cardiovascular diseases (28). Diabetes and other comorbidities may have a possible association to COVID-19 infection which showed progressive systemic injury in diabetic patients $(6,14)$.

\section{HYPERGLYCEMIA: A POTENTIAL RISK FACTOR}

Diabetes crucially potentiates COVID-19 development and adverse endpoints; thereby, well-controlled blood glucose, maintaining glycemic variation (range $3.9-10.0 \mathrm{mmol} / \mathrm{L}$ ), is associated with substantial decreases in adverse composite outcomes and death (29). Hyperglycemia is the potential risk factor for mortality and severity of SARS related diseases (30). It has been reported that hyperglycemia induced by diabetes linked to aggressive glycosylation and led to overproduction of advanced glycation end products (31). A study showed that 
aberrant glycosylation was associated with the dysfunction of immunoglobulin (32). Diabetes and SARS-CoV-2 coinfections can trigger stress and increase the secretion of hyperglycemia hormones, such as glucocorticoid and catecholamines, which results in increased blood glucose concentration and other diabetic complications (7). In Figure 1, we have summarized the potential mechanisms that explain the severity of COVID-19 in patients with diabetes comorbidity.

In brief, this manuscript shows some clue about SARS$\mathrm{CoV}-2$ infection in patients with diabetes comorbidity and further suggests that diabetes could potentiate the severity of

\section{REFERENCES}

1. WHO. Coronavirus Disease 2019 (COVID-19): Situation Report (Weekly Epidemiological Update) WHO website. (2020). Available online at: https:// www.who.int/publications/m/item/weekly-epidemiological-update--- 10 november-2020 (accessed November 13, 2020).

2. Zhu N, Zhang D, Wang W, Li X, Yang B, Song J, et al. A novel coronavirus from patients with pneumonia in China, 2019. N Engl J Med. (2020) 382:72733. doi: 10.1056/NEJMoa2001017

3. Guan WJ, Ni ZY, Hu Y, Liang WH, Ou CQ, He JX, et al. Clinical characteristics of coronavirus disease 2019 in China. N Engl J Med. (2020) 382:1708-20. doi: 10.1056/NEJMoa2002032

4. Yang X, Yu Y, Xu J, Shu H, Liu H, Wu Y, et al. Clinical course and outcomes of critically ill patients with SARS-CoV-2 pneumonia in Wuhan, China: a single-centered, retrospective, observational study. Lancet Respir Med. (2020) 5:475-81. doi: 10.1016/S2213-2600(20)30079-5

5. Zhang JJ, Dong X, Cao YY, Yuan YD, Yang YB, Yan YQ, et al. Clinical characteristics of 140 patients infected with SARS-CoV-2 in Wuhan, China. Allergy. (2020) 75:1730-41. doi: 10.1111/all.14238

6. Pranata R, Permana H, Huang I, Lim MA, Soetedjo NNM, Supriyadi R, et al. The use of renin angiotensin system inhibitor on mortality in patients with coronavirus disease 2019 (COVID-19): a systematic review and meta-analysis. Diabetes Metab Syndr. (2020) 14:983-90. doi: 10.1016/j.dsx.2020.06.047

7. Wang A, Zhao $\mathrm{W}, \mathrm{Xu} \mathrm{Z,} \mathrm{Gu} \mathrm{J.} \mathrm{Timely} \mathrm{blood} \mathrm{glucose} \mathrm{management} \mathrm{for} \mathrm{the}$ outbreak of 2019 novel coronavirus disease (COVID-19) is urgently needed. Diabetes Res Clin Pract. (2020) 162:108118. doi: 10.1016/j.diabres.2020.108118

8. Kulcsar KA, Coleman CM, Beck SE, Frieman MB. Comorbid diabetes results in immune dysregulation and enhanced disease severity following MERS-CoV infection. JCI Insight. (2019) 4:e131774. doi: 10.1172/jci.insight. 131774

9. Zhao Y, Zhao Z, Wang Y, Zhou Y, Ma Y, Zuo W. Single-cell RNA expression profiling of ACE2, thereceptor of SARS-CoV-2. Am J Respir Crit Care Med. (2020) 202:756-9. doi: 10.1164/rccm.202001-0179LE

10. Komukai K, Mochizuki S, Yoshimura M. Gender and the reninangiotensin-aldosterone system. Fundam Clin Pharmacol. (2010) 24:687-98. doi: 10.1111/j.1472-8206.2010.00854.x

11. Perico L, Benigni A, Remuzzi G. (2020). Should COVID-19 concern nephrologists? why and to what extent? The emerging impasse of angiotensin blockade. Nephron 144, 251-2.

12. Ding T, Zhang J, Wang T, Cui P, Chen Z, Jiang J, et al. Potential influence of menstrual status and sex hormones on female SARS-CoV- 2 infection: a crosssectional study from multicentre in Wuhan, China. Clin Infect Dis. (2020) ciaa1022. doi: 10.1093/cid/ciaa1022

13. Esler M, Esler D. Can angiotensin receptor-blocking drugs perhaps be harmful in the COVID-19 pandemic? J Hypertens. (2020) 38:781-2. doi: $10.1097 / \mathrm{HJH} .0000000000002450$

14. Wan Y, Shang J, Graham R, Baric RS, Li F. Receptor recognition by the novel coronavirus from Wuhan: an analysis based on decade-long structural studies of SARS coronavirus. J Virol. (2020) 94:e00127-0. doi: 10.1128/JVI.00127-20

15. Wrapp D, Wang N, Corbett KS, Goldsmith JA, Hsieh CL, Abiona O, et al. Cryo-EM structure of the 2019-nCoV spike in the prefusion conformation. Science. (2020) 367:1260-3. doi: 10.1126/science.abb2507
COVID-19 through various pathways which ultimately lead to advanced glycation end products, glucotoxicity, endothelial damage, vital organ damage, and death. These pathways might open a new avenue for future research and could employ to manage COVID-19 with diabetes comorbidity.

\section{AUTHOR CONTRIBUTIONS}

DS designed the concept. JSu and JSh drafted the manuscript. DS edited, finalized, and polished the manuscript. All authors approved the final manuscript.

16. Hoffmann M, Kleine-Weber H, Schroeder S, Krüger N, Herrler T, Erichsen $\mathrm{S}$, et al. SARS-CoV-2 cell entry depends on ACE2 and TMPRSS2 and is blocked by a clinically proven protease inhibitor. Cell. (2020) 181:271-80. doi: 10.1016/j.cell.2020.02.052

17. Lu R, Zhao X, Li J, Niu P, Yang B, Wu H, et al. Genomic characterisation and epidemiology of 2019 novel coronavirus: implications for virus origins and receptor binding. Lancet. (2020) 395:565-74. doi: 10.1016/S0140-6736(20)30251-8

18. Kuba K, Imai Y, Rao S, Gao H, Guo F, Guan B, et al. A crucial role of angiotensin converting enzyme 2 (ACE2) in SARS coronavirus-induced lung injury. Nature medicine, (2005) 11, 875-9. doi: 10.1038/nm1267

19. Iwata-Yoshikawa N, Okamura T, Shimizu Y, Hasegawa H, Takeda M, Nagata N. TMPRSS2 contributes to virus spread and immunopathology in the airways of murine models after coronavirus infection. J Virol. (2019) 93:e01815-8. doi: 10.1128/JVI.01815-18

20. Fang L, Karakiulakis G, Roth M. Are patients with hypertension and diabetes mellitus at increased risk for COVID-19 infection? Lancet Respir Med. (2020) 8:21. doi: 10.1016/S2213-2600(20)30116-8

21. Cure E, Cure MC. Angiotensin-converting enzyme inhibitors and angiotensin receptor blockers may be harmful in patients with diabetes during COVID-19 pandemic. Diabetes Metab Syndr. (2020) 14:349-50. doi: 10.1016/j.dsx.2020.04.019

22. Cheng H, Wang Y, Wang GQ. Organ-protective effect of angiotensinconverting enzyme 2 and its effect on the prognosis of COVID-19. J Med Virol. (2020) 92:726-30. doi: 10.1002/jmv.25785

23. Deisl C, Simonin A, Anderegg M, Albano G, Kovacs G, Ackermann D, et al. Sodium/hydrogen exchanger NHA2 is critical for insulin secretion in $\beta$-cells. Proc Natl Acad Sci. (2013) 110:10004-9. doi: 10.1073/pnas.1220009110

24. Channappanavar R, Perlman S. Pathogenic human coronavirus infections: causes and consequences of cytokine storm and immunopathology. In: Semin Immunopathol. (2017) 39:529-39. doi: 10.1007/s00281-017-0629-x

25. Huang C, Wang Y, Li X, Ren L, Zhao J, Hu Y, et al. Clinical features of patients infected with 2019 novel coronavirus in Wuhan, China. Lancet. (2020) 395:497-506. doi: 10.1016/S0140-6736(20)30183-5

26. Guo W, Li M, Dong Y, Zhou H, Zhang Z, Tian C, et al. Diabetes is a risk factor for the progression and prognosis of COVID-19. Diabetes Metab Res Rev. (2020) 36:e3319. doi: 10.1002/dmrr.3319

27. Tanaka $T$, Narazaki $M$, Kishimoto $T$. Immunotherapeutic implications of IL-6 blockade for cytokine storm. Immunotherapy. (2016) 8:959-70. doi: 10.2217/imt-2016-0020

28. Bruno G, Landi A, Barutta F, Ghezzo G, Baldin C, Spadafora L, et al. N-terminal probrain natriuretic peptide is a stronger predictor of cardiovascular mortality than C-reactive protein and albumin excretion rate in elderly patients with type 2 diabetes: the Casale Monferrato population-based study. Diabetes Care. (2013) 36:2677-82. doi: 10.2337/ dc13-0353

29. Zhu L, She ZG, Cheng X, Qin JJ, Zhang XJ, Cai J, et al. Association of blood glucose control and outcomes in patients with COVID-19 and pre-existing type 2 diabetes. Cell Metab. (2020) 31:1068-77.e3. doi: 10.1016/j.cmet.2020.04.021

30. Yang JK, Feng Y, Yuan MY, Yuan SY, Fu HJ, Wu BY,et al. Plasma glucose levels and diabetes are independent predictors for mortality 
and morbidity in patients with SARS. Diabet Med. (2006) 23:623-8. doi: $10.1111 /$ j.1464-5491.2006.01861.x

31. Erickson JR, Pereira L, Wang L, Han G, Ferguson A, Dao K, et al. Diabetic hyperglycaemia activates CaMKII and arrhythmias by Olinked glycosylation. Nature. (2013) 502:372-6. doi: 10.1038/nature 12537

32. Arnold JN, Wormald MR, Sim RB, Rudd PM, Dwek RA. The impact of glycosylation on the biological function and structure of human immunoglobulins. Annu Rev Immunol. (2007) 25:21-50. doi: 10.1146/annurev.immunol.25.022106.141702
Conflict of Interest: The authors declare that the research was conducted in the absence of any commercial or financial relationships that could be construed as a potential conflict of interest.

Copyright $(02021$ Sur, Sharma and Sharma. This is an open-access article distributed under the terms of the Creative Commons Attribution License (CC BY). The use, distribution or reproduction in other forums is permitted, provided the original author(s) and the copyright owner(s) are credited and that the original publication in this journal is cited, in accordance with accepted academic practice. No use, distribution or reproduction is permitted which does not comply with these terms. 